\title{
Propagation of Partial Discharge Signals in Stator Windings of Turbine Generators
}

\author{
A. J. M. Pemen, Member, IEEE, P. C. T. van der Laan, Member, IEEE, and Wout de Leeuw
}

\begin{abstract}
We studied the propagation of partial discharge pulses in a stator winding by means of measurements on a dismantled 35-MW generator and found that a stator winding acts as a transmission line. Therefore, a partial-discharge (PD) signal manifests itself at the generator terminals after a transit time that depends on the location of the discharge. Due to capacitive and inductive couplings in the end-winding region, a second propagation mode is present for higher frequencies. This "fast mode" manifests itself at the terminals without appreciable time delay. The capacitive and inductive couplings also cause crosstalk between the phases. As a consequence, a signal measured in one phase does not necessarily originate from a discharge in that phase. The amplitudes of the fast mode and, to a lesser extent, the slow mode (or transmission-line mode) are heavily damped when the discharge occurs further away from the measuring terminal. The consequence is that only discharges close to the measuring terminal can be observed. The propagation of a PD signal is not only influenced by the construction of the generator but also by its external connections.
\end{abstract}

Index Terms-High-voltage techniques, insulation testing, machine windings, partial discharges (PDs), turbogenerators.

\section{INTRODUCTION}

$\mathbf{F}$ OR a correct interpretation of partial-discharge (PD) measurements, we need to know how the actual electrical activity in a cavity is transferred to the measuring system. A commonly used engineering model for internal discharges, the $a b c$-model, is given in Fig. 1 [1].

A discharge causes a motion of charge $q_{c}$ within the cavity, capacitor $c$, resulting in a voltage drop at the terminals of the object. To detect the discharge, a coupling capacitor $C_{c}$ and a measuring impedance $Z_{m}$ are used. The measured apparent charge $q_{\text {app }}$ is related to the actual charge $q_{c}$ involved in the discharge according to $q_{\mathrm{app}} / q_{c}=b / c$. Calibration of the measuring circuit is possible by injection of a known charge into the terminals of the object under test (capacitors $a, b, c$ ).

For objects that cannot be regarded as lumped capacitors, the $a b c$ model is more complicated. Examples are gas-insulated substation (GIS) installations and high-voltage cables. A PD excites an electromagnetic wave that propagates along the GIS [2] or the cable. The object can no longer be regarded as

Manuscript received November 6, 2003; revised November 8, 2004. This work was supported in part by KEMA, Arnhem, The Netherlands, and in part by the Dutch Power Utilities EPON, EPZ, EZH, and UNA. Paper no. TEC-003192003.

A. J. M. Pemen and P. C. T. van der Laan are with the Eindhoven University of Technology, Electrical Power Systems Group, Eindhoven 5600 MB, The Netherlands (e-mail: a.j.m.pemen@tue.nl).

W. de Leeuw is with KEMA T\&D Power, Arnhem 6800 ET, The Netherlands (e-mail: w.de.leeuw@kema.nl).

Digital Object Identifier 10.1109/TEC.2005.847949 (a)

(b)

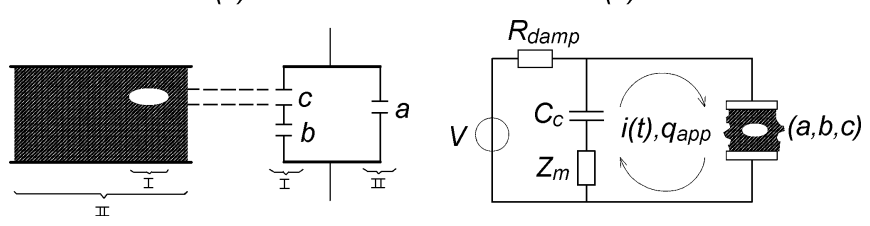

Fig. 1. (a) $A b c$ model and (b) standard measuring technique for PDs.

just a capacitance, but must also be seen as a transmission line. Calibration in PCs can be difficult due to damping and dispersion, caused by, for instance, semiconductive layers in cables or spacers in GIS. Reflections of the wave, for instance, at the cable ends can, however, be used to locate the discharge [3].

Also for complex electrical structures, such as transformer and stator windings, the $a b c$-model is not applicable. During the long path from the discharge site to the terminals, where the discharge can be measured, the signal is heavily distorted. For transformers, it is shown in [4]-[6] that the PD signal propagates in three modes: i) the higher frequencies $(0.1$ to $10 \mathrm{MHz})$ travel through the capacitive ladder network, formed by the interturn capacitances, ii) a traveling-wave mode (frequencies up to $10 \mathrm{kHz}$ ) follows the transmission line formed by the galvanic path of the winding, and iii) an oscillating component is determined by the internal resonant frequencies of the transformer $(10$ to $100 \mathrm{kHz}$ ). As a consequence, the amplitude and waveform of the response at the terminal strongly depends on the location of the discharge and on the design of the transformer.

Similar problems arise when measuring PDs in stator windings of turbine generators [7]. In 1983, Timperley showed that several types of incipient insulation failures generate travelling waves in a stator winding, and that their frequency characteristics depend on the origin of the defect [8]. Several authors report impulse response tests on stator windings; examples can be found in [9]-[14]. It is reported that a PD pulse essentially propagates as a travelling wave and that the stator winding can be regarded as a transmission line. This travelling-wave mode contains the lower frequency components. The higher frequencies are capacitively coupled through the winding and are heavily attenuated. In addition, [10] observes a strong crosstalk between the windings of the three phases. IEEE Standard 1434 [15] provides an overview of pulse propagation studies and the consequences for PD measurements and their calibration in terms of apparent charge.

This paper presents more experimental data on the propagation properties of a stator winding and the resulting consequences for discharge measurements. In experiments, pulses were injected at various places in the windings of an old 35-MW stator; the responses at the terminals were measured. In addition, 
(a)

(b)

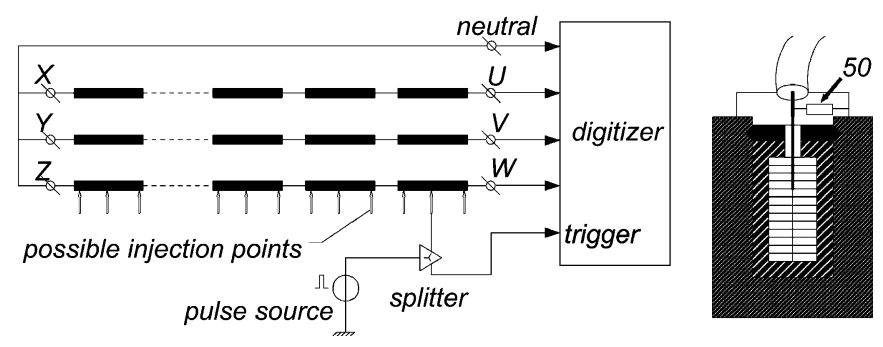

Fig. 2. (a) Experimental setup, and (b) injection point.

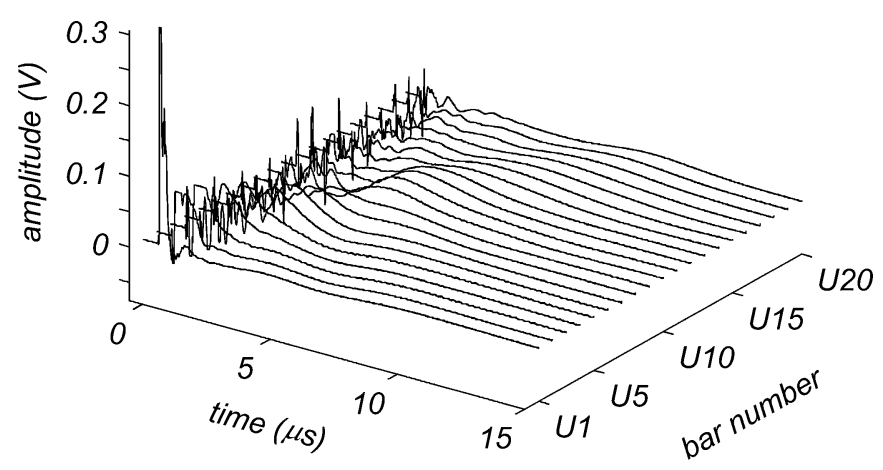

Fig. 3. Responses at the HV terminal of phase $\mathrm{U}$, when pulses are injected successively in the middle of each bar of this phase.

to illustrate the external propagation of PD signals, online measurements were carried out on a 125-MW turbine generator.

\section{EXPERIMENTAL SETUP}

To study the propagation of PD signals in a stator winding, we did experiments on the stator of an old 35-MW generator (rotor removed). The stator has 60 slots, with only one bar per slot. This is rather unusual; in general, large turbine generators have two bars per slot. The stator winding has three phases, with 20 bars per phase. We will characterize each bar with its phase and number. For instance, bar U9 is the ninth bar of phase $\mathrm{U}$ (looking from the high-voltage terminal). The experimental setup is sketched in Fig. 2.

A fast pulse (rise time $1 \mathrm{~ns}$, duration $50 \mathrm{~ns}$, amplitude $1 \mathrm{~V}$ ) was injected directly in the winding. A small hole was drilled in the insulation to connect the inner conductor of a coaxial cable directly to the conductor in the bar. The shield of the cable was carefully connected to the stator iron. To match the pulse generator as correctly as possible, a $50-\Omega$ resistor was installed in parallel at the injection point. The responses could be measured at the three high-voltage (HV) terminals and at the neutral terminal of the generator with $100-\mathrm{MHz}$ voltage probes. The injected pulse triggered the digitizer (Tektronix DSA602A) via a splitter. To prevent problems with interference, the digitizer was placed in an electromagnetic-compatibility (EMC) cabinet. The coaxial cable braids were solidly grounded at the EMC cabinet and at the measuring terminals.

\section{EXPERIMENTAL RESULTS}

Fig. 3 gives the responses at the high-voltage terminal of phase $U$, when pulses are injected successively in the middle

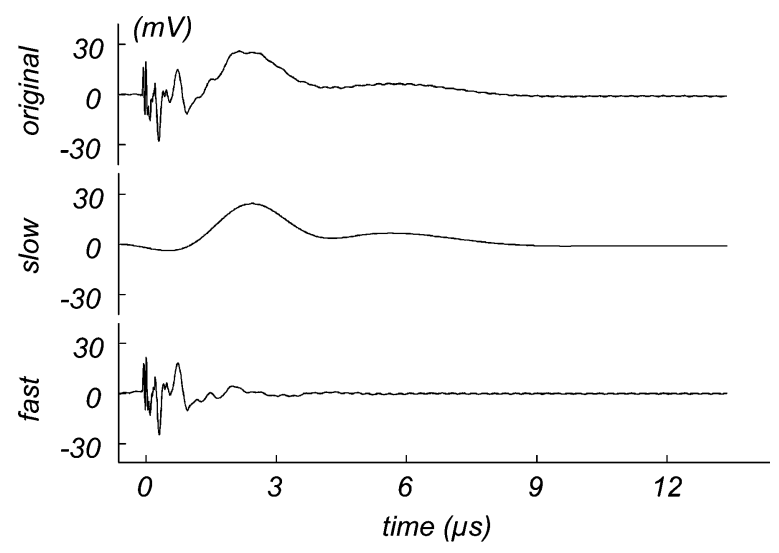

Fig. 4. Separation of the "slow mode" and the "fast mode." The original data are measured at the HV terminal of phase U, for pulse injection in bar U8. The slow mode is obtained by lowpass filtering of the original data. The fast mode is obtained after subtraction of the slow mode from the original data.

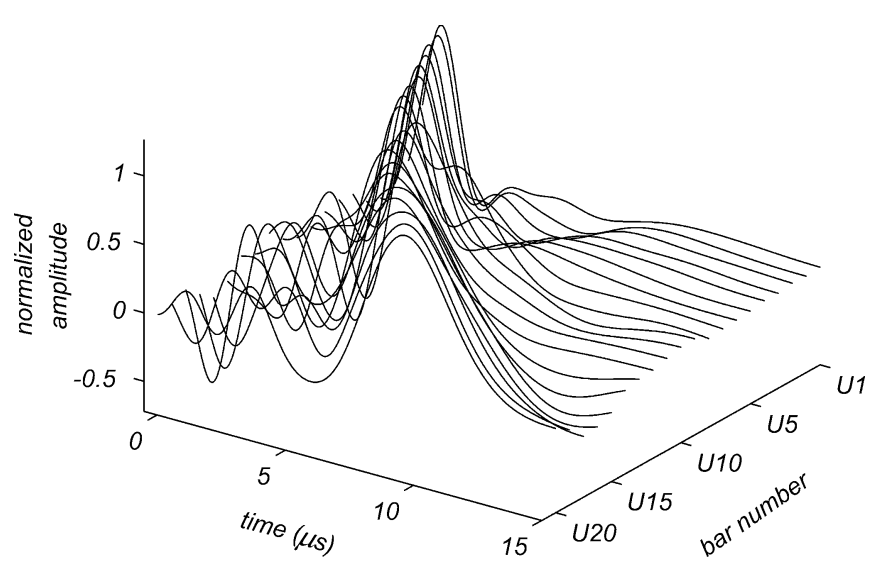

Fig. 5. Slow modes at the HV terminal of phase U, determined after digital lowpass filtering. Note that the order of the bar numbers is different from Fig. 3.

of each of the 20 bars of this phase. The bar number is given along one axis. Along the other two axes, the signal amplitude and time are given. We only discuss the results for phase U. The other two phases show similar results, which is plausible since the three phases are similar in geometry and construction.

As can be seen, the responses show two modes. A "fast mode" arrives at the terminals without a significant time delay and contains mainly the higher frequency components. The slow tails in Fig. 3 contain the lower frequency components, which apparently travel through the entire winding. This "slow mode" arrives at the terminals with a delay that depends on the place of injection.

\section{A. Analysis of the "Slow Mode" and the "Fast Mode"}

Both propagation modes can be separated by means of digital lowpass filtering. To obtain the slow mode, we used a fifth-order Butterworth filter with a cutoff frequency of $450 \mathrm{kHz}$. The fast mode is obtained by substraction of the slow mode from the original data. An example is given in Fig. 4.

Fig. 5 shows the slow modes for the responses at the highvoltage terminal of phase U. Note that the results are normalized; the maximum value of each waveform is set to one. It is clear from Fig. 5 that the slow mode travels through the entire winding before it arrives at the terminals of the generator. 


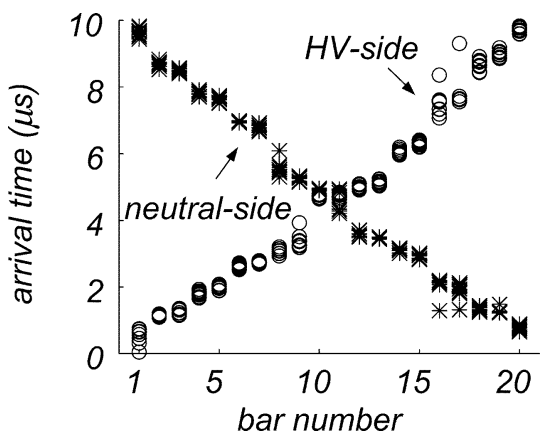

Fig. 6. Arrival times at the HV and neutral terminals of the slow modes. The figure shows all 180 measurements (the stator has 60 bars; measurements are done for injection at three positions in each bar).

The time of arrival depends on the place of injection as is shown in Fig. 6. At the time of arrival, we took the time of the maximum amplitude. Because the length of the end windings is different for various parts of the winding, the time of arrival is not fully linear with respect to the bar number. The transit time is $9.8 \mu \mathrm{s}$, for a winding length of $89 \mathrm{~m}$. Thus, the velocity of the slow mode is $9.1 \mathrm{~m} / \mu$ s or $3 \%$ of the speed of light.

The fast mode arrives at the terminals with a time delay varying from 59 to $86 \mathrm{~ns}$, as can be observed from Fig. 7. The arrival times depend on the place of injection and are given in Fig. 7(b) for all 180 measurements. The extra transit times $\Delta t_{1}$ and $\Delta t_{2}$ are equal for all measurements and are explained in Fig. 7(c). This figure shows three bars and their capacitive couplings at the end windings. Both the neutral terminal and the high-voltage terminal are at the same side of the generator (at the "beginning"). When pulses are injected at the beginning of a bar, the signal arrives at a terminal with a time delay of $59 \mathrm{~ns}$, partly caused by a delay in the measuring cable (16 ns) and by the transit time of the connection from the winding to the terminal. It is clear that when a pulse is injected at the end of a bar, it has to travel from "end" to "begin" before it can arrive at the terminals. The mean transit time in a bar is $28.2 \mathrm{~ns}$ for a bar length of $2.3 \mathrm{~m}$. Thus, the velocity of the fast mode is $81.6 \mathrm{~m} / \mu \mathrm{s}$, or $27 \%$ of the speed of light.

The velocity of the fast mode is determined by $c / \sqrt{ }\left(\varepsilon_{r} \mu_{r}\right)$, where $c$ is the speed of light, $\varepsilon_{r}$ is the relative dielectric constant (typically $\varepsilon_{r}$ is 4-6 for epoxy-mica insulation), and $\mu_{r}$ is the relative permeability. Assuming that the stator core behaves as a solid steel, the PD current flows in a thin sheet at the surface of the iron, determined by the skin depth $\delta$. Now we may regard the iron minus the skin layer as being free of the magnetic field. So most of the magnetic flux is present in the slot (thus within the dielectric with $\mu_{r}=1$ ). Apparently, the contribution of the magnetic field in the skin layer may not be ignored due to the large relative permeability of the iron $\left(\mu_{r}>1000\right)$, as can be seen from the relatively low velocity of the fast mode. The penetration of the magnetic field into a laminated core is analyzed by Tavner [16]. He concludes that the iron laminations themselves can carry the PD current. In the thin layer of insulation between the laminations, the current crosses over as a displacement current. As a consequence, the penetration of flux into the stator iron is larger than the skin depth; however, it is still small compared to the dimensions of the slots. (a)
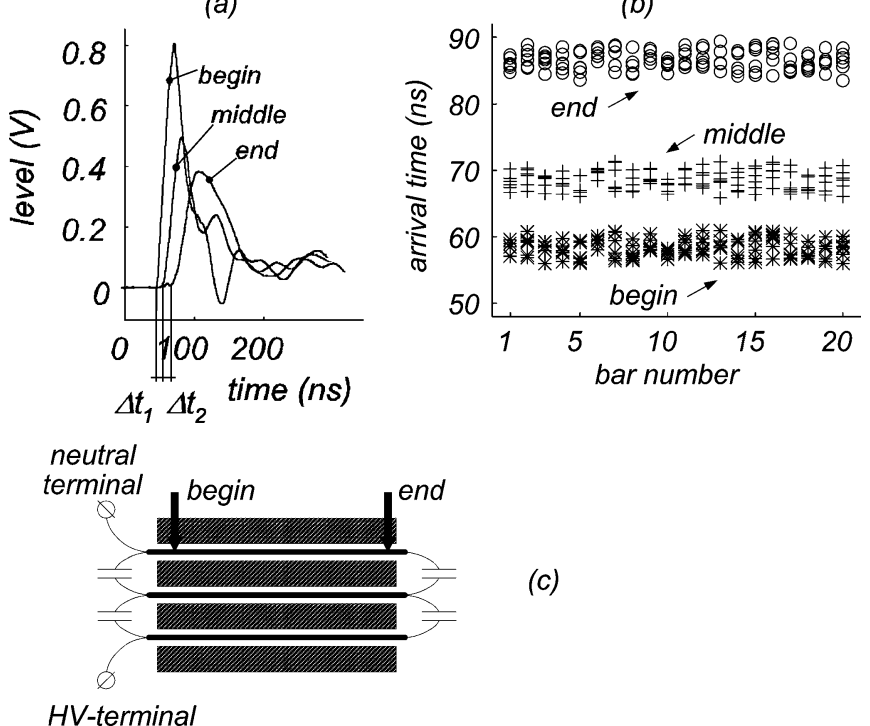

(c)

Fig. 7. (a) Responses at the HV terminal of phase $\mathrm{U}$ for pulse injection at three positions in bar U1. (b) Arrival times at the HV and neutral terminals of the fast modes for pulse injection at the beginning, middle, and end of all 60 bars, and (c) schematic representation of the propagation of the fast mode.

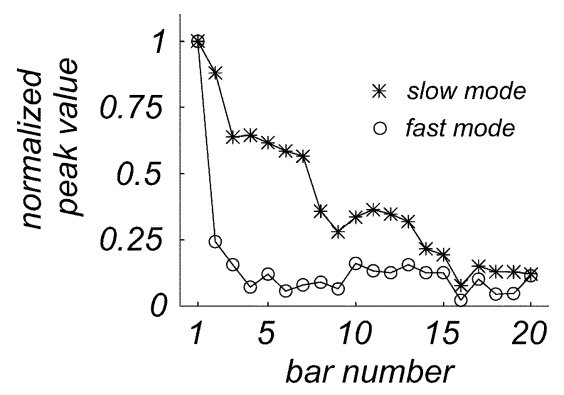

Fig. 8. Normalized peak values of the slow-mode $\left(^{*}\right)$ and the fast mode (o) when pulses are injected in each bar of phase $\mathrm{U}$ and measured at the $\mathrm{HV}$ terminal of this phase.

Fig. 8 gives the normalized peak values for the fast modes and the slow modes at the high-voltage terminal of phase $U$ (the peak values are normalized with reference to the largest value). The resulting amplitudes strongly depend on the place of injection and decrease when the pulse is injected further away from the measuring terminal. The higher frequencies (the fast mode) are especially heavily damped. At lower frequencies (the slow mode), about $35 \%$ of the winding can be seen with an attenuation of less than $50 \%$. For online measurements, most PDs occur in the high-voltage part of the winding. In that case, the rapid attenuation of the higher frequencies is not a serious disadvantage. Moreover, we prefer measurements at higher frequencies since, at lower frequencies, more interference is present.

These results show that a PD discharge signal follows a complicated path before it arrives at the generator terminals, where it can be measured. A stator winding acts as a transmission line, and a PD signal manifests itself at the generator terminals after a transit time that depends on the origin of the discharge. In addition, a second propagation mode is present for higher frequencies. This "fast mode" manifests itself at the terminals without appreciable time delay. The amplitudes of both the slow mode and the fast mode strongly depend on the origin of the discharge 
and decrease rapidly when the discharge occurs further away from the measuring terminal.

These results have been obtained on a relatively small generator (35 MW), with bar lengths of about $3 \mathrm{~m}$ and with only one bar in a slot. Generators with larger ratings have bar lengths from 5 to $7 \mathrm{~m}$, while two bars are present in one slot. So we must be careful to generalize the results. Nevertheless, both in [9] and [11], comparable results are reported for large 500-MW turbine generators. In both papers, the velocity of the slow mode is about $7 \mathrm{~m} / \mu \mathrm{s}$, which is close to our value $(9.1 \mathrm{~m} / \mu \mathrm{s})$. Both authors conclude that the attenuation is much larger for higher than for lower frequencies. This is not only for turbine generators, but also for hydro generators; comparable results were found and in [14], it is shown that the time delay between the fast mode and the slow mode can be used to locate a discharge. It is interesting to note that there is little difference in results, despite the huge differences in stator design.

\section{B. Charges Arriving at the Terminals}

Since the responses at the terminals (where the PDs are measured) depend on the origin of the PD, it is often stated that the apparent charge (in pC) of a discharge can only be determined if the place where the discharge occurs is known [17], [18]. This statement is based on measurements of the voltages at the terminals (as in Fig. 8). However, for PD measurements, the charge instead of the voltage at the terminals must be measured. To estimate the charge, the current that is measured at a terminal (the measured voltage divided by $50 \Omega$ ) is numerically integrated. The charges at all four measuring terminals when pulses are injected in each bar of phase $U$ are given in Fig. 9. Due to crosstalk between the phases, charge shows up at all four terminals. This crosstalk will be discussed later in this paper.

When the original recorded signals are numerically integrated (during $51.2 \mu \mathrm{s}$, including both the slow mode and the fast mode), the total charge at the four terminals depends not very much on the place of injection [Fig. 9(a)]. This would suggest that the measured apparent charge does not depend on the origin of the discharge if the complete response including all frequency components can be measured and integrated. However, for practical measurements, this is not possible. Standard discharge detectors only integrate frequencies above 100 to $500 \mathrm{kHz}$ (lower frequencies are rejected to suppress 50 or $60 \mathrm{~Hz}$ ). Detectors for measurements "in the field" operate at frequencies above $1 \mathrm{MHz}$ to suppress interference. Fig. 9(b) gives the charges when only the fast mode of the response is integrated. Now, the charges at the four terminals depend strongly on the place of injection and, compared to Fig. 9(a), only a fraction of the charges are observed. This shows that lower frequency components contribute most strongly to the apparent charge.

\section{Crosstalk Between the Phases}

We saw that the response at the terminals consists of two modes. For the slow mode, a stator winding acts as a transmission line. So the signals arrive at the generator terminals after a transit time that depends on the origin of the discharge. However, due to capacitive and inductive couplings, a second propagation mode (the fast mode) is present at higher frequencies. These couplings not only occur between bars of the same phase, (a)

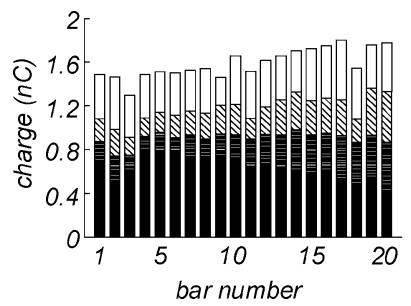

(b)

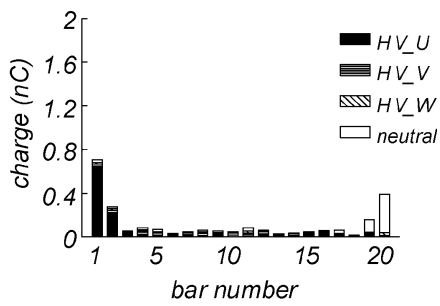

Fig. 9. Charges at all four terminals when pulses are injected in each bar of phase $U$. The charges are determined by numerical integration of (a) the complete responses (including both the fast and slow modes), and (b) only the fast modes.

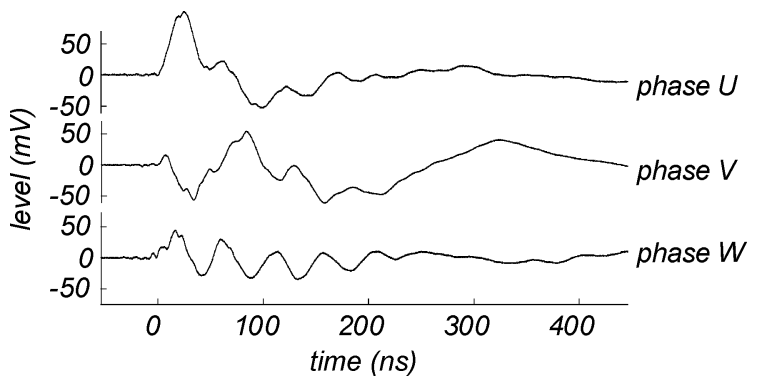

Fig. 10. Crosstalk between the phases: pulse injection in the 11th bar of phase $\mathrm{U}$, and the responses at the $\mathrm{HV}$ terminals of the three phases.

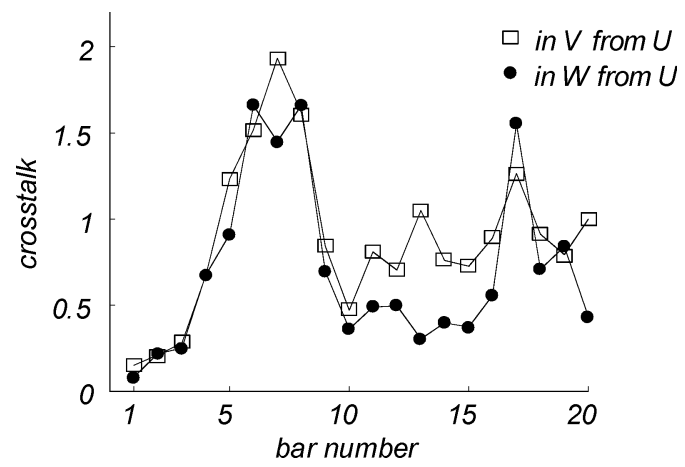

Fig. 11. Crosstalk from phase $\mathrm{U}$ to the other two phases.

but also cause crosstalk between the phases. This can be seen in Fig. 10, which shows the responses at the three high-voltage terminals when a pulse is injected in the 11th bar of phase U. Due to crosstalk, the signal shows up in each phase.

The crosstalk depends on the place of injection. This is shown in Fig. 11, which gives the crosstalk from phase $\mathrm{U}$ to the phases $\mathrm{V}$ and $\mathrm{W}$, when pulses are injected in each of the 20 bars of phase U. The crosstalk is defined as the ratio of the peak-topeak values, relative to phase U. For discharges in the first four bars (20\%) of the winding, the largest signal shows up at the terminal of the phase where the discharge occurs. In this case, the crosstalk is small and increases nearly linearly with the bar number. For discharges deeper into the winding, the crosstalk can be $>1$, which means that the largest signal does not show up in the phase where the discharge occurs, but in the other phases. Others have found even higher crosstalks [10]. We expect that the levels of crosstalk are related to the distribution of the stator bars over the slots. Some bars can have a coupling to a bar in a neighboring phase which is much closer to the terminal of 


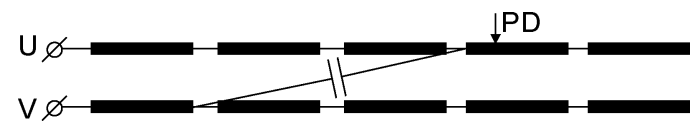

Fig. 12. Schematic representation of the crosstalk from bar number 4 of phase $\mathrm{U}$, to a bar of phase $\mathrm{V}$ which is much closer to the terminal.
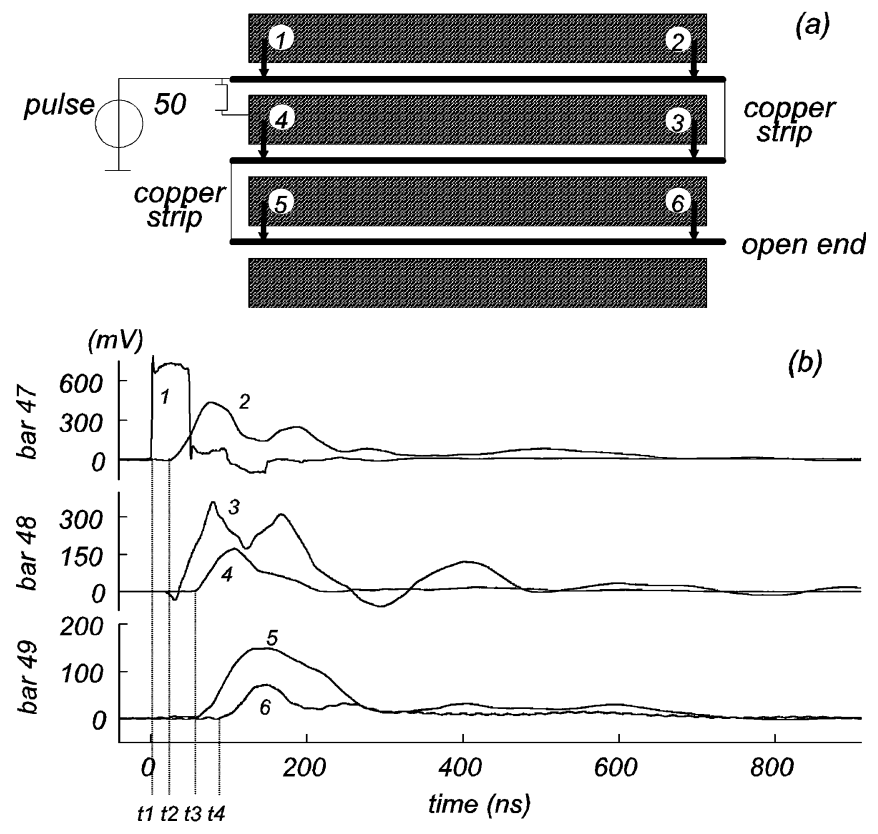

Fig. 13. (a) Setup for determination of capacitive or inductive couplings between three adjacent bars (end-windings removed), and (b) the measured responses at the six measuring points.

that phase. This is schematically shown in Fig. 12. Apparently, the signal in the neighboring phase will arrive at the terminal with much less attenuation and larger amplitude, as compared to the signal in the phase where the discharge occurs. In online measurements, only PDs from bars close to the high-voltage terminals occur; thus, it is plausible that the largest signal will show up in the phase where the discharge occurs. This enables a first localization of a PD; the phase where the discharge occurs can be determined.

The capacitive and inductive couplings between windings play an important role in the propagation of the PD signal through a stator winding. To determine whether these couplings take place in the slot-section or in the end-winding region, we removed the end windings for three adjacent bars. The bars were connected in series by means of short copper strips [Fig. 13(a)]. A pulse was injected at the beginning of the first bar. The responses were measured at two positions in each bar (points 1 to 6) and are given in Fig. 13(b).

The injected pulse propagates along the three bars in a transmission-line mode and without any capacitive or inductive couplings between the three bars. The transit times in a single bar vary between 28 and 32 ns and correspond with the results for the fast mode [Fig. 7(b)]. Apparently no coupling takes place in the slot sections, so the earlier described coupling occurs in the end windings.

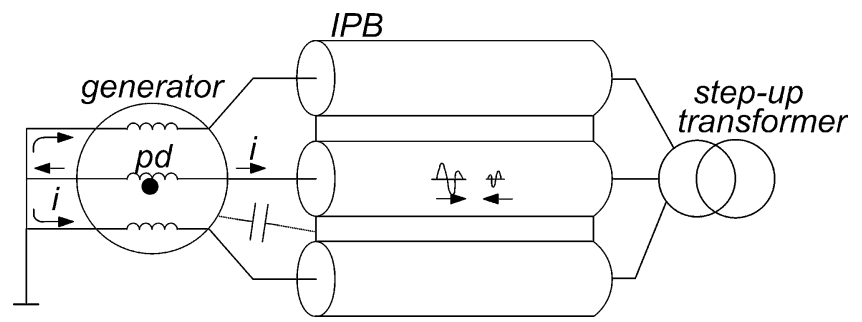

Fig. 14. Propagation of external PD signals.

\section{EXTERNAL PROPAGATION OF PD SigNALS}

PDs cause traveling waves in the stator windings. Results in the previous section show that the propagation of these waves inside the generator is strongly influenced by the capacitive and inductive coupling between the windings. Due to this coupling, the signals propagate in two modes and, in addition, crosstalk to other phases occurs. A heavily distorted fraction of the PD signal manifests itself at the generator terminals, where it can be measured.

Not only does the interior of the generator influence the PD signal, but so does the exterior. This is shown schematically in Fig. 14. On the high-voltage side, the external PD signal propagates along the isolated phase bus (IPB), which forms a good quality transmission line. At the step-up transformer, the signal reflects back toward the generator. The circuit is closed via the large parasitic capacitance between the IPB and the generator. Due to crosstalk inside the generator, signals will also show up in the other two phases, where they propagate in a similar manner.

Part of the PD signal manifests itself at the neutral side, mainly through capacitive and inductive coupling inside the generator. Now, the external circuit is closed via the other two phases. Consequently, there is not only crosstalk inside the generator but also outside it. The sum of the three neutral currents tends to be zero and, therefore, the current through the neutral grounding connection is only small.

\section{ONLINE Measurements ON a 125-MW Generator}

The external propagation of PD signals will be illustrated by measurements carried out on a 125-MW turbine generator. Six 200-MHz inductive current sensors were installed around the three neutral and the three high-voltage terminals of the generator. In addition, in each phase, two 500-pF/100-MHz capacitive sensors have been installed in the isolated-phase bus (IPB); one close to the generator and one near the step-up transformer. Details about the inductive and capacitive sensors can be found in [7]. The sensor signals are recorded by means of a digital oscilloscope (Tektronix DSA602A).

A first measurement, performed during regular operation of the generator, is shown in Fig. 15. Instead of a discharge, a pulse with a pulsewidth of $10 \mathrm{~ns}$ and an amplitude of $1.5 \mathrm{~V}$ [Fig. 15(a)] is injected at the capacitive sensor at the transformer side of phase $\mathrm{U}$ and is measured by means of the three capacitive sensors at the generator side [Fig. 15(b)], the inductive sensors at the three high-voltage terminals [Fig. 15(c)] and the three inductive sensors near the neutral terminal [Fig. 15(d)]. Note: the different transit times of the signals are caused by the transit 


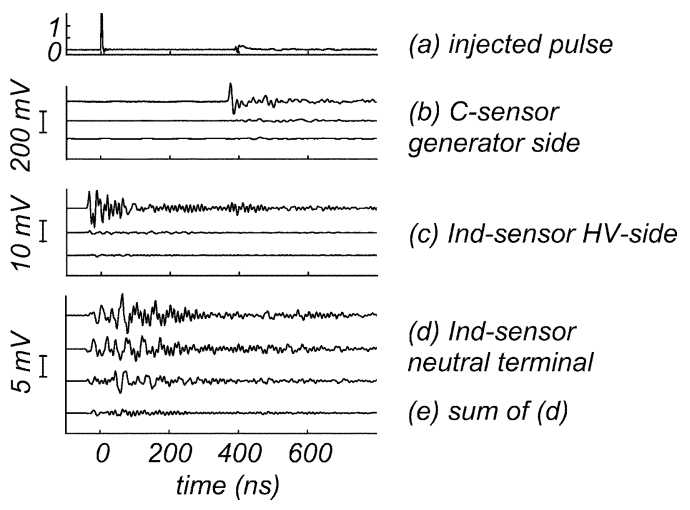

Fig. 15. Propagation of a pulse injected at the capacitive sensor at the transformer side of phase $\mathrm{U}(\mathrm{a})$, and measured by the capacitive sensors at the $\mathrm{HV}$-side (b), the inductive sensors at the HV-terminals (c), and the inductive sensors at the neutral terminals (d). Note that the transit times are not corrected for the different lengths of measuring cables.

times along the IPB and by different lengths of measuring cables. Since the latter are unfortunately unknown, it is not possible to analyze the transit times.

At the high-voltage side, the injected signal shows up at both the capacitive sensor and the inductive sensor of phase U. Due to the differentiating character of both sensors, bipolar pulses are measured. No significant signal is present in the other two phases, which shows that no crosstalk takes place in the IPB or near the generator terminals. The sum of the currents as measured by the inductive sensors [Fig. 15(c)] is not zero; thus, the circuit must be closed via a parasitic capacitance between the IPB and the generator. A small fraction of the injected signal shows up at the three neutral terminals of the generator [Fig. 15(d)]. Since the signals show no clear pulse and arrive simultaneously at the three neutral terminals, we assume that they are caused by capacitive and inductive coupling inside the generator. The calculated sum of the three currents is nearly zero [Fig. 15(e)], which confirms that the neutral grounding connection carries hardly any PD current.

The external propagation of a true PD signal, measured during regular operation of the generator, is shown in Fig. 16. We assume that this discharge occurs close to the high-voltage terminals of phase $\mathrm{U}$, because the crosstalk to the phases $\mathrm{V}$ and $\mathrm{W}$ is small [Fig. 16(c)]. Nevertheless, a small fraction of the signal shows up at the neutral side [Fig. 16(a)]. The signals at the neutral side are probably caused by capacitive and inductive couplings between the windings, since no clear pulse can be recognized and the signals show up without significant transit time. Because the sum of the three external neutral currents is about zero, the "external circuit" must be closed via the other two phases. As a consequence, a signal measured at the neutral terminal of one phase does not necessarily belong to a PD in that phase. Since the sum of the three external neutral current is about zero, it is expected that the neutral grounding connection carries no or hardly any current. However, PD measurement using a current probe installed at the grounding connection has been applied for many years [8].

At the high-voltage side, the PD manifests itself at the generator terminals and propagates with the speed of light along the IPB. The sum of the currents as measured with the three cur-

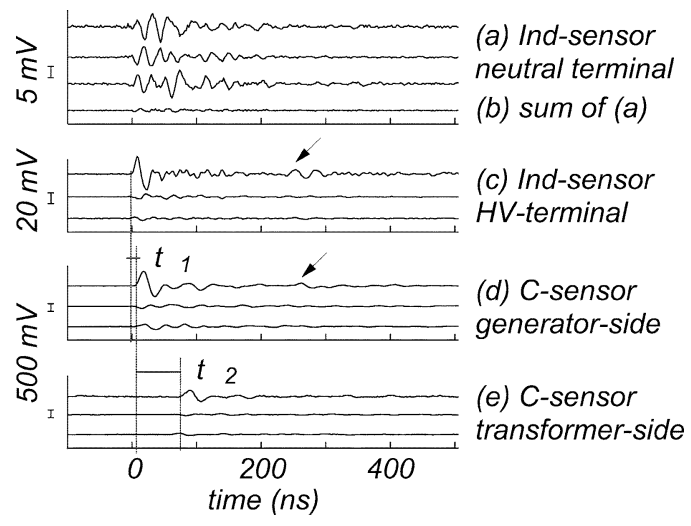

Fig. 16. Partial discharge in phase $\mathrm{U}$, measured by the inductive sensors at the neutral side (a), the inductive sensors at the HV terminals (c), the capacitive sensors at the generator side (d), and the capacitive sensors at the transformer side (e).

rent sensors at the high-voltage terminals is not zero. Thus, the external circuit must close via a parasitic capacitance between generator and IPB, as in Fig. 14.

The transit-time $\Delta t_{1}$ is $7 \mathrm{~ns}$ for all three phases and corresponds with the distance $(\approx 2 \mathrm{~m})$ between the inductive sensors at the generator terminals and the capacitive sensors at the generator side. The transit-time $\Delta t_{2}$ is $70 \mathrm{~ns}$ for phase $\mathrm{U}, 56 \mathrm{~ns}$ for phase $\mathrm{V}$, and $66 \mathrm{~ns}$ for phase $\mathrm{W}$, and again corresponds with the distance $(\approx 20 \mathrm{~m})$ between the capacitive sensors at the generator and at the step-up transformer side. At the step-up transformer, the signal reflects back toward the generator. The reflected signals are indicated with arrows. The reflection time as measured with the inductive sensors is 252 ns and corresponds with twice the transit time along the IPB $(\approx 76 \mathrm{~m})$. The reflection time as indicated for the capacitive sensor at the generator side is $238 \mathrm{~ns}$ and corresponds with twice the transit time between the sensor and the step-up transformer $(\approx 72 \mathrm{~m})$. The transit times between various sensors and reflections of the PD signals can be used to verify whether PD activity takes place inside the generator or, for instance, near the step-up transformer. In case of this $125-\mathrm{MW}$ generator, all recorded PD signals originated from the generator. No discharges from other sources could be detected.

Due to crosstalk inside the generator, at the high-voltage side, the PD in phase U shows up in all three phases. In the case of Fig. 16, this crosstalk is small, which is an indication that the discharge occurs close to the high-voltage terminal of phase $\mathrm{U}$. The signals caused by the crosstalk propagate in a similar manner as the PD signal in phase $\mathrm{U}$.

\section{CONCLUSION}

We studied the propagation of PD pulses in a stator winding and found that, for lower frequencies, a stator winding acts as a transmission line. Therefore, a PD signal manifests itself at the generator terminals after a transit time that depends on the location of the PD.

Due to capacitive and inductive couplings in the end-winding region, a second propagation mode is present for higher frequencies. This "fast mode" manifests itself at the terminals without appreciable time delay. 
The capacitive and inductive couplings cause crosstalk between the phases. As a consequence, a signal measured in one phase does not necessarily originate from a discharge in that phase.

The amplitudes of the fast mode and, to a lesser extent, the slow mode are heavily damped when the discharge occurs further away from the measuring terminal. The consequence is that only discharges close to the measuring terminal can be observed, which is expected not to be a serious disadvantage.

The total charge at the terminals, if the responses are integrated over their full frequency range, is only weakly dependent on the origin of the discharge. However, in practice, the detection bandwidth is limited. Thus, it is impossible to obtain the total apparent charge and whatever is measured at the machine terminals is dependent on the origin of the discharge.

The propagation of a PD signal is not only influenced by the interior of the generator but also by its external connections. At the high-voltage side, the PD signal propagates along the IPB and reflects at the step-up transformer. The signal path is closed via the large parasitic capacitance between the IPB and the generator. The signals in the neighboring phases, caused by crosstalk inside the generator, propagate in a similar manner. At the neutral side, the PD signal path is closed via the other two phases. As a consequence, a signal measured at one of the neutral terminals does not necessarily originate from a PD in that phase.

\section{REFERENCES}

[1] F. H. Kreuger, Partial Discharge Detection in High-Voltage Equipment. London, U.K.: Butterworth \& Co., 1989.

[2] PD Detection Sensitivity and E-M Wave Attenuation in a $400 \mathrm{kV}$ GIS Sub-Station. Bedford, MA, Aug. 1997.

[3] P. C. J. M. van der Wielen, E. F. Steennis, and P. A. A. F. Wouters, "Fundamental aspects of excitation and propagation of on-line partial discharge signals in three-phase medium voltage cable systems," IEEE Trans. Dielectr. Electr. Insul., vol. 10, no. 4, pp. 678-688, Aug. 2003.

[4] A. T. Thoeng, "Detection and location of partial discharges in power transformers," Holectechniek, vol. 3, pp. 52-57, 1973.

[5] R. E. James, F. E. Trick, B. T. Phung, and P. A. White, "Interpretation of pd quantities as measured at the terminals of HV power transformers," IEEE Trans. Electr. Insul., vol. EI-21, no. 4, pp. 629-638, Aug. 1986.

[6] J. Fuhr et al., "Detection and location of internal defects in the insulation of power transformers," IEEE Trans. Electr. Insul., vol. 28, no. 6, pp. 1057-1067, Dec. 1993.

[7] A. J. M. Pemen, "Detection of Partial Discharges in Stator Windings of Turbine Generators," Ph.D. thesis, Dept. Elect. Eng., Eindhoven University of Technology, 2000.

[8] J. E. Timperley, "Detection of insulation deterioration through electrical spectrum analysis," in Proc. 16th Electrical/Electronics Insulation Conf., 1983, paper IEEE 83CH1952-1/83/0000-0060, pp. 60-64.

[9] A. Wilson, R. J. Jackson, and N. Wang, "Discharge detection techniques for stator windings," Proc. Inst. Elect. Eng. B, vol. 132, no. 5, pp. 234-244, Sep. 1985.

[10] M. Henriksen, G. C. Stone, and M. Kurtz, "Propagation of partial discharge and noise pulses in turbine generators," IEEE Trans. Energy Convers., vol. EC-1, no. 3, pp. 161-166, Sep. 1986.

[11] R. E. James, B. T. Thung, and R. Miller, "The effect of end-winding configurations on the transmission of steep pulses through high voltage stator windings," in Proc. 5th Int. Symp. High-Voltage Engineering, Braunschweig, Germany, Aug. 24-28, 1986, paper 93.02.

[12] R. E. James, B. T. Phung, and Q. Su, "Investigation of a low frequency partial discharge location method utilizing a $500 \mathrm{MW} / 22 \mathrm{kV}$ turbo-generator stator," in Proc. IEEE Int. Symp. Electrical Insulation, Toronto, ON, Canada, Jun. 3-6, 1990, pp. 100-105.

[13] J. W. Wood, H. G. Sedding, W. K. Hogg, I. J. Kemp, and H. Zhu, "Partial discharges in HV machines; initial considerations for a PD specification," Proc. Inst. Elect. Eng. A, vol. 140, no. 5, pp. 409-416, Sep. 1993.
[14] J. W. L. Simpson, R. C. Tychsen, Q. Su, T. R. Blackburn, and R. E. James, "Evaluation of partial discharge detection techniques on hydrogenerators in the Australian snowy-mountains scheme Tumut-1 case study," IEEE Trans. Energy Convers., vol. 10, no. 1, pp. 18-24, Mar. 1995.

[15] Trial-Use Guide to the Measurement of Partial Discharges in Machinery, 2000.

[16] P. J. Tavner and R. J. Jackson, "Coupling of discharge currents between conductors of electrical machines owing to laminated steel core," Proc. Inst. Elect. Eng. B, vol. 135, no. 6, pp. 295-307, Nov. 1988

[17] I. J. Kemp, H. Zhu, H. G. Sedding, J. W. Wood, and W. K. Hogg, "Toward a new partial discharge calibration strategy based on the transfer function of machine stator windings," Proc. Inst. Elect. Eng., Sci., Meas., Technol., vol. 143, no. 1, pp. 57-62, Jan. 1996.

[18] H. Zhu, "Analysis of partial discharge calibration difficulties in HV rotating machines," in Proc. 10th Int. Symp. High-Voltage Engineering, Montreal, QC, Canada, Aug. 25-29, 1997.

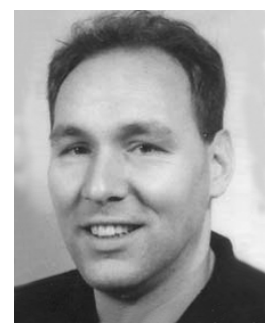

A. J. M. Pemen (M'98) was born in Breda, The Netherlands, in 1961. He received the B.Sc. degree in electrical engineering from the College of Advanced Technology, Breda, in 1986, and the Ph.D degree in electrical engineering from Eindhoven University of Technology, Eindhoven, The Netherlands, in 2000

Before joining the group Electrical Power Systems of the Eindhoven University of Technology in 1998 as Assistant Professor, he worked for KEMA T\&D Power, Arnhem, The Netherlands. He is currently involved in research on pulsed power and pulsed plasma. His research interests include high-voltage engineering, pulsed power, plasmas, and renewable energy systems. Among his achievements are the development of an online monitoring system for PDs in turbine generators, a 30-kW pulsed-corona system for industrial applications, and a pulsed-corona tar cracker. He is the founder of the Dutch Generator Expertise-Center, Arnhem, The Netherlands.

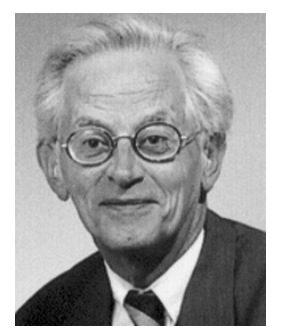

P. C. T. van der Laan (M'78) was born in 1935. He received the Ph.D. degree from Utrecht University, Utrecht, The Netherlands, in 1964.

He studied technical physics at Delft University, Delft, The Netherlands. In 1959, he joined the new FOM-Institute for Plasma Physics, Jutphaas, The Netherlands. He did research on plasmas confined by magnetic fields over a period of 19 years on rapidly compressed "pinches." This research was partially done at the University of Wisconsin in Madison; one year at the Massachusetts Institute of Technology, Cambridge; and three years at the Los Alamos Scientific Laboratory, Los Alamos, NM. In 1978, he became Full Professor of Power Engineering in the Department of Electrical Engineering, Eindhoven University of Technology, with an emphasis on high-voltage discharges, measuring techniques, and electromagnetic compatibility (EMC).

Dr. van der Laan is a member of the American Physical Society.

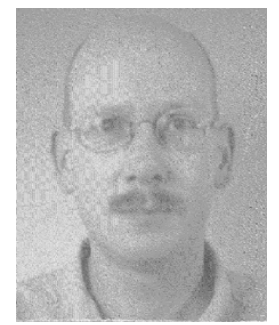

Wout de Leeuw was born in Utrecht, The Netherlands, in 1965 . He received the B.Sc. degree in electrical engineering from the College of Advanced Technology, Zwolle, The Netherlands, in 1989.

In 1989, he was a Technical Assistant in an Electrical Research Department with KEMA T\&D. He is now especially involved in expertise centers for transformers and rotating electrical machines. A considerable part of his work has been PD measurements on generators. 